\title{
ORIGINAL
}

\section{ECMO for severe ARDS: systematic review and individual patient data meta-analysis}

\author{
Alain Combes ${ }^{1,2^{*}}$ (D) Giles J. Peek ${ }^{3}$, David Hajage ${ }^{4}$, Pollyanna Hardy ${ }^{5}$, Darryl Abrams ${ }^{6,7}$, Matthieu Schmidt ${ }^{1,2}$,
} Agnès Dechartres ${ }^{4}$ and Diana Elbourne ${ }^{8}$

(ㄷ) 2020 Springer-Verlag GmbH Germany, part of Springer Nature

\begin{abstract}
Purpose: To assess the effect of venovenous extracorporeal membrane oxygenation (ECMO) compared to conventional management in patients with severe acute respiratory distress syndrome (ARDS).

Methods: We conducted a systematic review and individual patient data meta-analysis of randomised controlled trials (RCTs) performed after Jan 1, 2000 comparing ECMO to conventional management in patients with severe ARDS.

The primary outcome was 90 -day mortality. Primary analysis was by intent-to-treat.

Results: We identified two RCTs (CESAR and EOLIA) and combined data from 429 patients. On day 90,77 of the 214 (36\%) ECMO-group and 103 of the 215 (48\%) control group patients had died (relative risk (RR), 0.75, 95\% confidence interval $\left.(\mathrm{Cl}) 0.6-0.94 ; P=0.013 ; I^{2}=0 \%\right)$. In the per-protocol and as-treated analyses the RRs were $0.75(95 \% \mathrm{Cl}$ $0.6-0.94)$ and 0.86 (95\% Cl 0.68-1.09), respectively. Rescue ECMO was used for 36 (17\%) of the 215 control patients (35 in EOLIA and 1 in CESAR). The RR of 90-day treatment failure, defined as death for the ECMO-group and death or crossover to ECMO for the control group was 0.65 (95\% Cl 0.52-0.8; $\left.1^{2}=0 \%\right)$. Patients randomised to ECMO had more days alive out of the ICU and without respiratory, cardiovascular, renal and neurological failure. The only significant treatment-covariate interaction in subgroups was lower mortality with ECMO in patients with two or less organs failing at randomization.
\end{abstract}

Conclusions: In this meta-analysis of individual patient data in severe ARDS, 90-day mortality was significantly lowered by ECMO compared with conventional management.

Keywords: Extracorporeal membrane oxygenation, Acute respiratory distress syndrome, Adult patients, Individual patient data meta-analysis

*Correspondence: alain.combes@aphp.fr

1 Sorbonne Université, INSERM, UMRS_1166-ICAN, Institute of Cardiometabolism and Nutrition, 75013 Paris, France

Full author information is available at the end of the article

\section{Introduction}

Ventilatory management of patients with severe acute respiratory distress syndrome (ARDS) has improved over the last decades with a strategy combining low tidal volume (VT) ventilation [1], high positive end-expiratory pressure (PEEP) [2, 3], neuromuscular blocking agents [4] and prone positioning [5]. However, ventilator-induced lung injury (VILI) may persist in these patients since a recent and large epidemiological study showed that their hospital mortality was still 46\% [6]. Recently, even higher mortality was reported for patients with severe acute 
respiratory syndrome coronavirus2 (SARS-CoV-2) infection who needed invasive mechanical ventilation [7-9].

Venovenous extracorporeal membrane oxygenation (ECMO) providing full blood oxygenation, $\mathrm{CO}_{2}$ elimination and combined with more gentle ventilation has benefited from major technological advances in the last 15 years [10, 11]. In 2009, favourable outcomes were reported in patients who received ECMO during the influenza A (H1N1) pandemic [12-14]. The Conventional Ventilator Support vs Extracorporeal Membrane Oxygenation for Severe Acute Respiratory Failure (CESAR) trial $[15,16]$ showed that transfer to an ECMO centre was associated with fewer deaths or severe disabilities at 6 months compared with conventional mechanical ventilation $(37 \%$ vs. $53 \% ; p 0=0.03)$, although 6 month mortality was not significantly reduced ( $37 \%$ vs. $45 \%$; $p=0.07$ ). The more recent ECMO to Rescue Lung Injury in Severe ARDS (EOLIA) trial showed a non-statistically significant reduction in 60-day mortality with ECMO (35\% vs. $46 \% ; p=0.09$ ) [17]. However, neither trial was separately powered to detect a $10-15 \%$ survival benefit with ECMO.

We performed a systematic review with an individual patient data meta-analysis of randomised controlled trials comparing ECMO to conventional mechanical ventilation in patients with severe ARDS. The primary objective was to evaluate the effect of ECMO on 90-day mortality. Secondary objectives included the evaluation of ECMO for other clinical outcomes and in pre-specified subgroups for the primary outcome.

\section{Methods}

\section{Study design}

This systematic review and meta-analysis followed the Preferred Reporting Items for Systematic reviews and Meta-Analyses for Individual Patient Data (PRISMA-IPD checklist in eTable 1 in the Supplement) and the protocol was registered in PROSPERO (CRD42019130034) on May 1st 2019.

\section{Eligibility criteria}

We included all randomised controlled trials (RCTs) evaluating venovenous ECMO in the experimental group and conventional ventilatory management in the control group, that included patients with ARDS fulfilling the American-European Consensus Conference definition [18] or the Berlin definition for ARDS [19], and that were published or whose primary completion date was after 2000 [10, 20, 21]. This choice was justified by the major improvements in intensive care treatments and in ECMO technology that occurred in the last two decades. Additional information on selection criteria is provided in the Supplement.

\section{Take home message}

In this meta-analysis of individual patient data in severe ARDS, 90-day mortality was significantly lowered by ECMO compared with conventional management. Patients randomised to ECMO had more days alive out of the ICU and without respiratory, cardiovascular, renal and neurological failure

\section{Search strategy}

We searched MEDLINE via PubMed, EMBASE and the Cochrane Central Register of Controlled Trials (Central) from January 1, 2000 to September 30, 2019 using a search algorithm developed for the purpose of this study and adapted to each database (eTable 2 in the Supplement). We also searched trial registries including ClinicalTrials.gov and the International Clinical Trial Registry Platform (ICTRP) for completed and ongoing trials, conference proceedings of major critical care societies and screened reference lists of identified articles as well as systematic or narrative reviews on the topic (see the Supplement).

\section{Selection and data collection}

Selection was conducted independently by two reviewers (DA and MS) on titles and abstracts first and then, on the full text. For each included RCT, the corresponding author was contacted to provide fully anonymized individual patient data as well as format, coding and definition of any variables. Risk of bias in each trial was evaluated by two independent reviewers (DH and $\mathrm{AD}$ ) using the updated version of the risk-of-bias tool developed by Cochrane [22] (see the Supplement).

\section{Study outcomes}

The primary endpoint was mortality 90 days after randomisation. Main secondary endpoints comprised time to death up to 90 days after randomisation, treatment failure up to 90 days, defined as crossover to ECMO or death for patients in the control group, and death for patients in the ECMO group, number of days alive and out-of-hospital between randomisation and day 90, number of days alive without mechanical ventilation, renal replacement therapy and vasopressor support between randomisation and day 90 . Other preplanned secondary outcomes comprised mortality at 28 and 60 days after randomisation, number of days alive and out of the ICU between randomisation and day 90, number of days alive without respiratory failure, neurological failure, cardiovascular failure, liver failure, renal failure and coagulation failure, defined as the corresponding component sequential organ failure assessment (SOFA) score greater 
than 2 between randomisation and day 90. Data related to patients' management, causes of death and safety outcomes were also described (see the Supplement).

\section{Statistical analysis}

The statistical analysis was performed for each outcome of interest using individual patient data. An intentionto-treat analysis was used for all outcomes, whereby all patients were analysed in the groups to which they were randomised. The measures of treatment effect were risk ratios for binary outcomes, hazard ratios for time-toevent outcomes and mean differences for quantitative outcomes. The primary endpoint was defined as a binary outcome and analysed using both one-step (as primary analysis) and two-steps (as sensitivity analysis) methods [23]. In the one-step method, we analysed both studies simultaneously to obtain the combined treatment effect with $95 \%$ CIs and $p$-value using a generalized linear mixed effect model to account for the clustering of data within each trial with a random effect. In the two steps method, we first analysed separately each trial using individual patient data before combining them using a random effects meta-analysis model to account for variability between studies. A two-step method was used for all secondary outcomes. Heterogeneity was evaluated with the Cochran's Q-test, $\mathrm{I}^{2}$ statistic and between study variance $\tau^{2}$. Survival curves for the time to death up to 90 days were generated using individual patient data and the Kaplan-Meier method.

We conducted sensitivity analyses for the primary outcome in different populations (per-protocol, as-treated). The per-protocol population included all randomised patients having received the treatment attributed by randomisation (i.e., patients having received ECMO in the ECMO arm and patients not having received ECMO in the control arm). The as-treated population compared patients receiving ECMO to those who did not receive ECMO, whatever the randomisation arm. A sensitivity analysis excluding trials at high risk of bias was also planned.

We explored whether the effect of ECMO on 90-day mortality varied according to baseline patient characteristics (see the Supplement). For each subgroup, the treatment-subgroup interaction was tested in the one-step model. For quantitative baseline characteristics, we used the median values to define the subgroups. All these subgroup analyses were pre-planned.

Alpha risk was set at 5\% for the primary outcome. For all secondary outcomes, we did not correct for multiple testing. As such, subgroup and sensitivity analyses should be considered as exploratory. All the analyses were performed with the use of $R$ software version 3.6.1 ( $R$ Foundation).
The quality of evidence for the seven most important outcomes was graded with GRADEpro GDT (GRADEpro GDT: GRADEpro Guideline Development Tool [Software]; McMaster University, 2015 (developed by Evidence Prime, Inc.; Available from gradepro.org).

\section{Results}

\section{Selection process and general characteristics}

From the 1179 references identified by the search strategy, we included two randomised controlled trials fulfilling our eligibility criteria-CESAR and EOLIA $[15,17]$. Reasons for exclusion are reported in eFig. 1 of the Supplement. The two trials provided individual patient data for all randomised patients (429 overall, 180 in CESAR and 249 in EOLIA), and there was no eligible trial not providing individual patient data. Detailed characteristics of the two trials are reported in eTable 3 in the Supplement.

Comparison of patient characteristics at randomisation did not show baseline imbalance between groups (Table 1 and eTables 4 and 5 in the Supplement). The main disorder leading to study entry was severe hypoxia (in $88 \%$ of the patients, with a mean $\left( \pm\right.$ SD) $\mathrm{PaO}_{2} / \mathrm{FiO}_{2}$ of $75 \pm 34 \mathrm{~mm} \mathrm{Hg}$ ). The main cause of ARDS was pneumonia ( $>60 \%$ of the patients) and $39 \%$ had 3 or more organs failing at randomisation. Of the 214 patients randomised to the ECMO groups, 189 (88\%) received ECMO (98\% and $76 \%$ in EOLIA and CESAR, respectively). Rescue extracorporeal gas exchange was used for $36(17 \%)$ of the 215 control patients ( 35 patients crossed over to ECMO in EOLIA, and 1 to pumpless arteriovenous $\mathrm{CO}_{2}$ removal in CESAR that was a protocol violation by the conventional management team as rescue extracorporeal gas exchange was not part of the CESAR trial design). Risk of bias was judged low in both trials (eFigure 2 in the Supplement).

\section{Primary outcome}

By day 90, 77 (36\%) ECMO-group and 103 (48\%) control group patients had died (relative risk, $0.75,95 \%$ confidence interval $0.6-0.94 ; p=0.013$ ) (Table 2 and Fig. 1). Results were similar in the one-step and two-steps models. There was no evidence of heterogeneity across studies $\left(p=0.64, I^{2}=0 \%, \tau^{2}=0.000\right)$.

\section{Secondary outcomes}

The hazard ratio for death within 90 days after randomisation in the ECMO group, as compared with the control group, was 0.65 (95\% CI 0.49-0.88) (Fig. 2). The relative risk of treatment failure, defined as death by day 90 for the ECMO-group and death or crossover to ECMO for the control group was 0.65 (0.52-0.8) (Table 2 and eFig. 3 in the Supplement). At 90 days, ECMO-group patients 
Table 1 Characteristics of the patients at randomisation

\begin{tabular}{|c|c|c|}
\hline Characteristic & $\begin{array}{l}\text { ECMO } \\
\text { group } \\
(N=214)\end{array}$ & $\begin{array}{l}\text { Control } \\
\text { group } \\
(N=215)\end{array}$ \\
\hline Age, years & $46.6 \pm 15.2$ & $48.3 \pm 14.8$ \\
\hline Male-no. (\%) & $138(65)$ & $143(67)$ \\
\hline $\begin{array}{l}\text { Median (interquartile) time since intuba- } \\
\text { tion, } \mathrm{h}\end{array}$ & 35 [16-95] & $36[16-100]$ \\
\hline \multicolumn{3}{|l|}{ ARDS aetiology-no. (\%) } \\
\hline Pneumonia & $136(64)$ & $131(61)$ \\
\hline Other & $78(36)$ & $84(39)$ \\
\hline 3 or more organs failed ${ }^{a}$ & $82(38)$ & $84(39)$ \\
\hline Predicted mortality ${ }^{b}$ & $0.34 \pm 0.23$ & $0.34 \pm 0.22$ \\
\hline $\mathrm{PaO}_{2} / \mathrm{FiO}_{2}$ & $76 \pm 35$ & $75 \pm 33$ \\
\hline $\mathrm{pH}$ & $7.30 \pm 0.37$ & $7.26 \pm 0.24$ \\
\hline \multicolumn{3}{|l|}{ Disorder leading to study entry } \\
\hline Hypoxia & $184(86 \%)$ & $192(89 \%)$ \\
\hline Uncompensated hypercapnia & $30(14 \%)$ & $23(11 \%)$ \\
\hline PEEP, $\mathrm{cm} \mathrm{H}_{2} \mathrm{O}$ & $12.3 \pm 6.8$ & $12.7 \pm 6.8$ \\
\hline Respiratory system compliance, $\mathrm{ml} / \mathrm{cm} \mathrm{H}_{2} \mathrm{O}$ & $25.8 \pm 11.8$ & $25.3 \pm 8.8$ \\
\hline Murray score & $3.3 \pm 0.6$ & $3.3 \pm 0.4$ \\
\hline Chest radiograph (quadrants infiltrated) & $3.4 \pm 0.9$ & $3.5 \pm 0.8$ \\
\hline
\end{tabular}

Plus-minus values are means \pm SD; see eTable 5 the Supplement for missing data

ECMO denotes extracorporeal membrane oxygenation, ARDS the acute respiratory distress syndrome, $\mathrm{PaO}_{2}$ partial pressure of arterial oxygen, $\mathrm{FiO}_{2}$ the fraction of inspired oxygen, $\mathrm{PaO}_{2} / \mathrm{FiO}_{2}$ the ratio of the partial pressure of arterial oxygen to the fraction of inspired oxygen, PEEP positive end-expiratory pressure Missing data were $<3 \%$ for patients' characteristics at randomisation, except for predicted mortality, respiratory system compliance and Murray score (see eTable 5 in the Supplement)

a Number of organ failed (0-6) defined as the corresponding component sequential organ failure assessment (SOFA) score $>2$

b APACHE2 (CESAR) and SAPS2 (EOLIA) scores were both translated to predicted probability of ICU mortality

had more days alive without ventilation (40 vs 31 days, mean difference, 8 days; $95 \%$ CI 2-15) and out of the ICU (36 vs 28 days, mean difference, 8 days; 95\% CI 2-14) than those in the control group (Table 2 and eFig. 4 in the Supplement).

At day 60 post-randomisation (90-day follow-up was not available for the following outcomes in EOLIA), patients in the ECMO group had more days alive without vasopressors ( 35 vs 28 days, mean difference, 8 days; $95 \%$ CI, 3 to 13), renal replacement therapy (35 vs 28 days, mean difference, 7 days; 95\% CI 2-13) and neurological failure (38 vs 31 days, mean difference, 7 days; $95 \%$ CI 2-13) than those in the control group (Table 2 and eFig. 5 in the Supplement). Prone positioning and low-volume low-pressure mechanical ventilation were applied to $71 \%$ and $85 \%$ of control group patients, respectively (Table 3 ). Multiorgan failure and respiratory failure were the main causes of death in both groups (Table 3), while a cannulation-related fatal complication occurred in 3 of the 225 patients who received ECMO. Of the 214 patients randomised to ECMO, 7 (3\%) died before ECMO could be established. Additional data on secondary outcomes are provided in Tables 2 and 3 and eFig. 6 in the Supplement.

\section{Sensitivity and subgroup analyses}

The relative risks of death at day 90 post-randomisation according to the per-protocol and as-treated analyses were 0.75 (95\% CI 0.6-0.94) and 0.86 (95\% CI 0.68-1.09), respectively (eFig. 7 in the Supplement). The only significant treatment-covariate interaction identified in subgroup analyses was the number of organs failing at randomisation with $\mathrm{RR}=0.53$ (95\% CI 0.36-0.78) among patients with $1-2$ organ failures and $R R=1.00(95 \% \mathrm{CI}$ $0.78-1.3$ ) among patients with 3 or more organ failures, $p=0.006$ for interaction (Fig. 3). There was no evidence to suggest a differential treatment effect for any other subgroups.

\section{Quality of evidence}

The Summary Of Findings Table reporting the evaluation of the quality of evidence for the seven most important outcomes is presented in eTable 6 in the Supplement. The level of evidence was high for mortality at 90 days, time to death and treatment failure.

\section{Discussion}

In this individual patient data meta-analysis of patients with severe ARDS included in the CESAR [15] and EOLIA [17] randomised trials, there is strong evidence to suggest that early recourse to ECMO leads to a reduction in 90-day mortality and less treatment failure compared with conventional ventilatory support. Patients randomised to ECMO also had more days alive out of the ICU and without respiratory, cardiovascular, renal and neurological failure.

The benefit of ECMO in severe ARDS patients has long been debated [24-27]. Because of highly challenging design and conduct issues, only four randomised trials of extracorporeal life support for adult patients with acute respiratory failure have been performed in the last 5 decades [15, 17, 28, 29]. Our meta-analysis included only the two most recent trials (CESAR [15] and EOLIA [17]) since major advances in ICU care and in ECMO techniques have occurred in the past 15 years making the two older trials not relevant for comparison [10, 20, 21]. In addition the two older trials did not use venovenous ECMO. One used venoarterial ECMO [28] and one used low-flow veno-venous extracorporeal $\mathrm{CO}_{2}$ removal [29]. Characteristics of patients included in EOLIA and CESAR were comparable regarding ARDS aetiology and disease severity at randomisation. Patients were enrolled early after the initiation of invasive mechanical 
Table 2 Endpoints

\begin{tabular}{|c|c|c|c|c|c|}
\hline Endpoint & $\begin{array}{l}\text { ECMO group } \\
(N=214)\end{array}$ & $\begin{array}{l}\text { Control group } \\
(N=215)\end{array}$ & $\begin{array}{l}\text { Relative Risk or } \\
\text { difference }(95 \% \mathrm{Cl})\end{array}$ & $p$ value & $I^{2}(\%)$ \\
\hline \multicolumn{6}{|l|}{ Primary endpoint } \\
\hline Day 90 mortality—no. (\%) & $77(36)$ & $103(48)$ & $0.75(0.6-0.94)$ & 0.013 & 0 \\
\hline \multicolumn{6}{|l|}{ Secondary endpoints ${ }^{a}$} \\
\hline Day 90 treatment failure-no. (\%) & $77(36)$ & $119(55)$ & $0.65(0.52-0.8)$ & & 0 \\
\hline Day 28 mortality—no. (\%) & $50(23)$ & $88(41)$ & $0.57(0.4-0.81)$ & & 33 \\
\hline Day 60 mortality—no. (\%) & $73(34)$ & $101(47)$ & $0.73(0.58-0.92)$ & & 0 \\
\hline Day 1-90 ICU-free days ${ }^{b}$ & $36 \pm 32$ & $28 \pm 33$ & $8(2-14)$ & & 0 \\
\hline Day 1-90 hospital-free days ${ }^{b}$ & $22 \pm 27$ & $18 \pm 27$ & $4(-1-9)$ & & 0 \\
\hline Day 1-90 ventilation-free days ${ }^{\mathrm{b}}$ & $40 \pm 35$ & $31 \pm 34$ & $8(2-15)$ & & 0 \\
\hline Day 1-60 vasopressor-free days $s^{b, c}$ & $35 \pm 26$ & $28 \pm 27$ & $8(3-13)$ & & 0 \\
\hline Day 1-60 RRT-free days ${ }^{b, c}$ & $35 \pm 27$ & $28 \pm 27$ & $7(2-13)$ & & 0 \\
\hline Day 1-60 neurological failure-free days $s^{b, c, d}$ & $38 \pm 28$ & $31 \pm 30$ & $7(2-13)$ & & 6 \\
\hline
\end{tabular}

Data are mean (SD) or number (\%)

ECMO denotes extracorporeal membrane oxygenation, ICU intensive care unit and RRT renal replacement therapy

a The width of confidence intervals have not been adjusted for multiplicity and should not be used to infer definitive treatment differences

${ }^{b}$ Free-days were calculated assigning zero free-days to patients who died during the follow-up period

c Day-by-day follow-up was limited to Day 60 in the EOLIA trial

$d$ Neurological failure was defined by the number of days without neurological depression requiring system monitoring/support' in CESAR study and the neurologic component of the sequential organ failure assessment (SOFA) score greater than 2

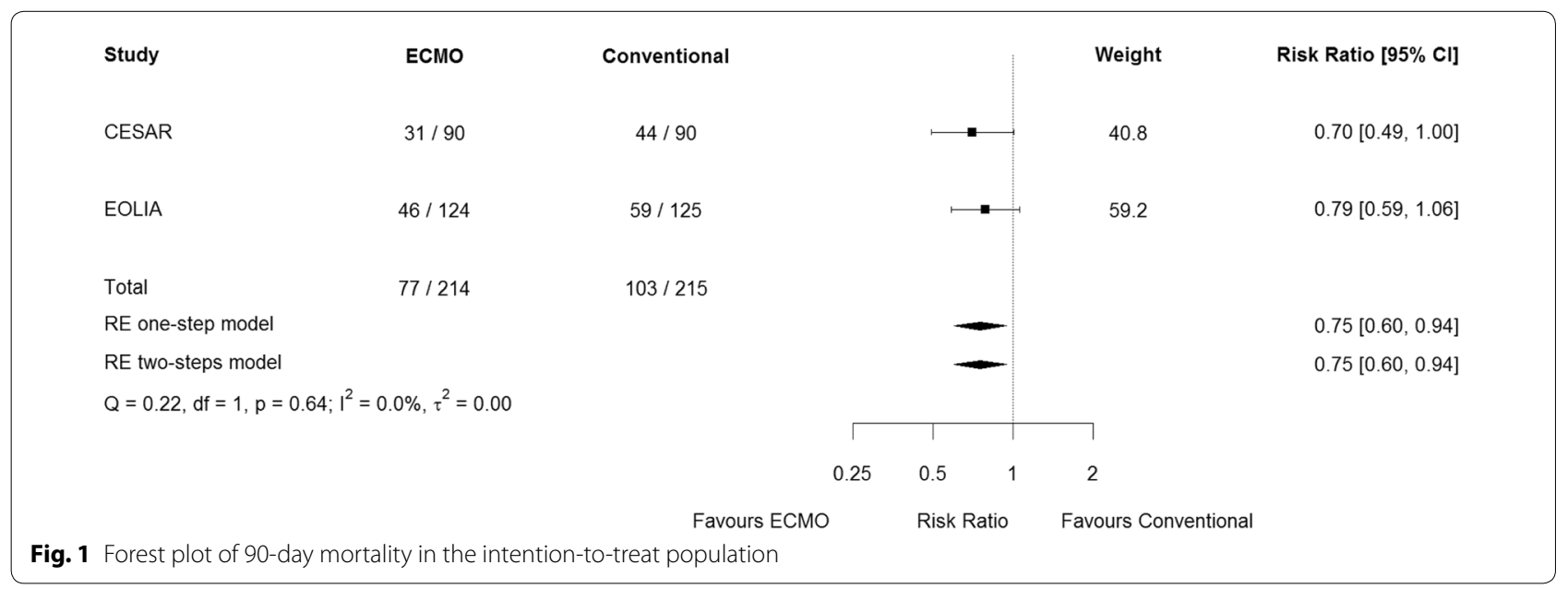

ventilation and rates of control patients being proned and receiving low-volume low-pressure mechanical ventilation were high. Both EOLIA and CESAR trials showed a comparable survival benefit with ECMO, but neither was individually powered to detect a reasonable survival difference between groups. Specifically, the data safety monitoring board of EOLIA, following pre-specified guidance using a sequential design with a two-sided triangular test based on 60-day mortality, recommended stopping the trial for futility after $75 \%$ of the maximal sample size had been enrolled, because the probability of demonstrating a $20 \%$ absolute risk reduction in mortality with ECMO was considered unlikely. Our meta-analysis, which includes a much larger number of patients and shows higher survival with ECMO in both the intention-to-treat and per-protocol analyses provides strong evidence about the benefit of ECMO in severe ARDS. Our results also extend the conclusions of a post-hoc Bayesian analysis of EOLIA indicating a very high probability of ECMO success in severe ARDS patients, ranging from 88 to $99 \%$ depending on the chosen priors [30]. Our results are consistent with two previous aggregated data meta-analyses in the field: one was a network meta-analysis considering different interventions whose impact is limited by the 


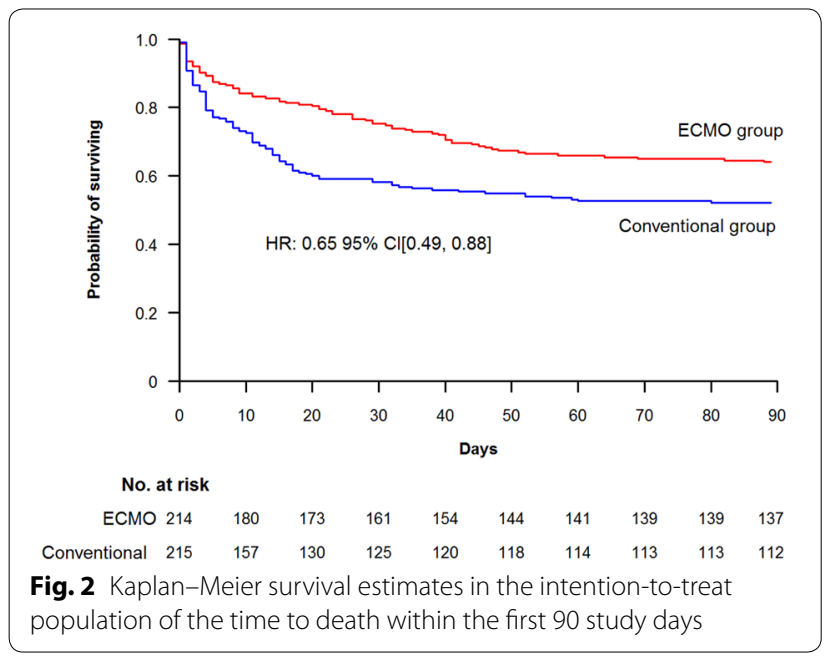

lack of direct comparisons [31] and the other focused on ECMO [32]. Our IPD meta-analyses goes beyond these two previous studies and provides a stronger evidence on the benefit of ECMO in ARDS for the following reasons.

Table 3 Patients' management and other outcomes

\begin{tabular}{|c|c|c|}
\hline Endpoint & $\begin{array}{l}\text { ECMO } \\
\text { group } \\
(N=214)\end{array}$ & $\begin{array}{l}\text { Control } \\
\text { group } \\
(N=215)\end{array}$ \\
\hline Received ECMO—no. (\%) & $189(88)$ & $36(17)$ \\
\hline Days under $\mathrm{ECMO}^{\mathrm{a}}$ & $14.3 \pm 12.6$ & $16.6 \pm 15$ \\
\hline Received LVLP MV—no. $(\%)^{\mathrm{b}}$ & $205(98)$ & $181(85)$ \\
\hline $\begin{array}{l}\text { Prone position (before and after ran- } \\
\text { domisation)—no. }(\%)^{b}\end{array}$ & $114(54)$ & $151(71)$ \\
\hline iNO or prostacyclin-no. $(\%)^{b}$ & $84(40)$ & $110(51)$ \\
\hline Renal replacement therapy-no. $(\%)^{b}$ & $106(50)$ & $129(60)$ \\
\hline Steroids-no. $(\%)^{b}$ & $156(74)$ & $140(65)$ \\
\hline ICU length of stay, days & $29.7 \pm 24.6$ & $23.6 \pm 35.9$ \\
\hline For survivors & $35.2 \pm 22.5$ & $39.5 \pm 26.3$ \\
\hline For non-survivors & $20.2 \pm 17.6$ & $15.4 \pm 16.2$ \\
\hline Hospital length of stay, days & $49 \pm 43.1$ & $42.7 \pm 69.3$ \\
\hline For survivors & $58.3 \pm 23.8$ & $60 \pm 28.5$ \\
\hline For non-survivors & $20.2 \pm 17.6$ & $15.4 \pm 16.2$ \\
\hline \multicolumn{3}{|l|}{ Cause of death } \\
\hline Respiratory failure & $13(6)$ & $36(17)$ \\
\hline Multiple organ failure & $35(16)$ & $44(20)$ \\
\hline ECMO cannulation-related & $2(1)$ & $1(0)$ \\
\hline Miscellaneous & $27(13)$ & $22(10)$ \\
\hline
\end{tabular}

Data are mean (SD) or number (\%); see eTable 5 in the Supplement for missing data

ECMO denotes extracorporeal membrane oxygenation, LVLP MV, low-volume low-pressure mechanical ventilation, iNO inhaled nitric oxide, and ICU intensive care unit

Missing data were $<2.5 \%$ for patients' outcomes (see eTable 5 in the

Supplement)

${ }^{\text {a }}$ For patients who received ECMO

${ }^{b}$ From randomisation to day 60
IPD meta-analyses provides a higher level of evidence than aggregated data meta-analyses, because they are independent of the quality of reporting in included studies and allow evaluation of other important outcomes such as time to death and number of days without organ failures [33, 34].

In this study, we showed that, beyond mortality, duration and severity of organ failures also favoured ECMO, and these results were highly consistent between the two studies. This observation provides insights into the potential pathophysiological mechanisms of ECMOassociated benefits in severe ARDS [10]. Although extracorporeal gas exchange may rescue some patients dying of profound hypoxemia or in whom high pressure mechanical ventilation has become dangerous, minimization of lung stress and strain associated with positive pressure ventilation may drive most of the improved outcomes observed under ECMO [10]. Ultraprotective ventilation with very low VTs, driving pressures and respiratory rates [35], and, therefore, minimized overall mechanical power transmitted to lung alveoli [36] may reduce ventilator-induced lung injury, pulmonary and systemic inflammation and ultimately organ failure leading to death. These data also reinforce the recent recommendation of the World Health Organization (WHO) [37], and the Surviving Sepsis Campaign [38] to consider ECMO support in coronavirus disease 2019 (COVID19)-related ARDS with refractory hypoxemia if lung protective mechanical ventilation was insufficient to support the patient [39].

Meta-analyses of individual patient data can also explore outcomes in important subgroups and suggest which population may derive the greatest benefit of a specific intervention, which is very limited in aggregated data meta-analyses [40]. In this study, the mortality of patients with only one or two organs failing at randomisation was almost halved with ECMO (22\% vs. $41 \%$ ), while it was not substantially different between groups in patients with $\geq 3$ organ failures. This finding suggests that veno-venous ECMO may not be able to improve the outcomes of ARDS patients with severe shock and multiple organ failure. In EOLIA, patients with baseline $\mathrm{PaO}_{2} /$ $\mathrm{FiO}_{2}>66 \mathrm{mmHg}$ or those enrolled due to severe respiratory acidosis and hypercapnia, seemed to derive the greatest benefit of ECMO [17].

This analysis has several limitations. First, inclusion criteria were more stringent for the EOLIA trial, in which, for example, ventilator optimization ( $\mathrm{FiO} 2>80 \%$, VT at $6 \mathrm{ml} / \mathrm{kg}$ predicted body weight and PEEP $>10 \mathrm{~cm} \mathrm{H}_{2} \mathrm{O}$ ) was mandatory before enrolment. However, it should be noted that baseline patient characteristics were comparable regarding ARDS severity at inclusion (eTable 4 in the Supplement). 


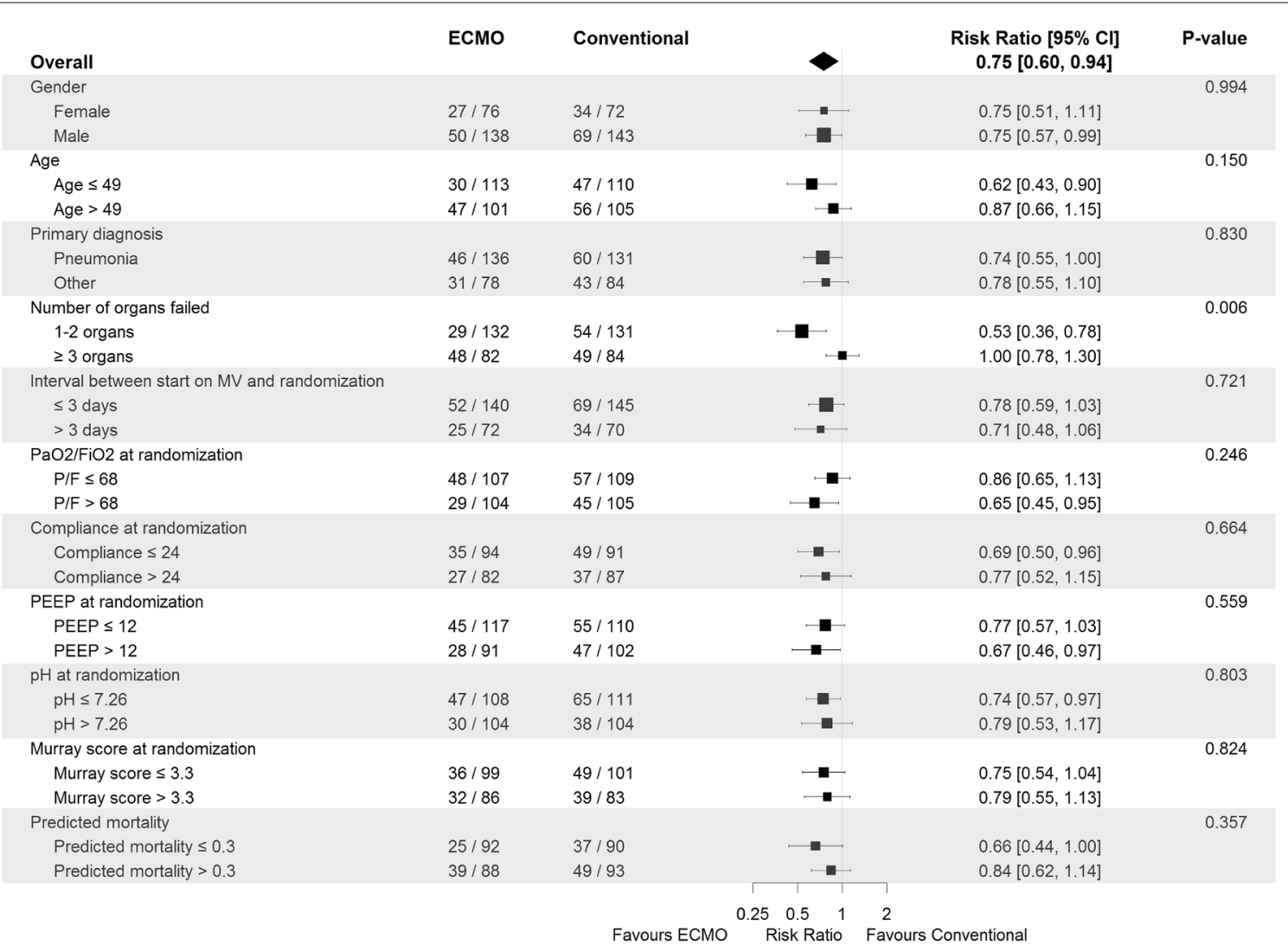

Fig. 3 Subgroup analyses for the primary outcome according to baseline characteristics. MV, mechanical ventilation; number of organ failed (0-6) defined as the corresponding component sequential organ failure assessment (SOFA) score > 2; APACHE2 (CESAR) and SAPS2 (EOLIA) scores were both translated to predicted probability of ICU mortality

Second patient management was not similar in the two studies. In CESAR, $24 \%$ of patients randomised to the ECMO arm did not receive ECMO and there was no standardized protocol for mechanical ventilation in the control group. Conversely, in EOLIA, 98\% of patients randomised to ECMO received the intervention, the mechanical ventilation strategy in the control group followed a strict protocol, and rescue ECMO was applied to $28 \%$ of control group patients who had developed refractory hypoxemia. However, this meta-analysis showed a significantly lower mortality with ECMO in the per-protocol analysis including only patients in whom ECMO had been initiated in the ECMO arm and patients not having ECMO in the control arm. This analysis minimizes the aforementioned management differences, since the least severe patients who did not receive ECMO after MV optimization in CESAR were excluded from the ECMO arm and the most severe patients who needed rescue ECMO in EOLIA were excluded from the control arm. In contrast, ECMO was not associated with a mortality benefit in the as-treated population, but such an analysis strongly disadvantages the ECMO group, which includes the most severe control patients rescued by ECMO. Second, this meta-analysis does not provide detailed data on ECMO-related safety endpoints, since they were not reported in CESAR. Death directly related to ECMO cannulation was rare in both studies and the rates of stroke and major bleeding were also low in EOLIA, in which a restrictive anticoagulation strategy was applied [17]. Third, no long-term outcomes beyond 90 day post-randomisation were analysed although the CESAR trial [15] and a retrospective cohort of ARDS patients [41] reported satisfactory long-term health-related quality-of-life after ECMO. Fourth, only the CESAR trial provided a cost-effectiveness analysis that suggested a benefit of the transfer of ARDS patients to a centre with an ECMO-based management protocol [15]. Our results, showing improved survival, with more days alive out of the ICU and without the need for major organ support are in line with CESAR's cost-effectiveness data. Fifth, many conditions such as MV duration $>7$ days prior to ECMO or major comorbidities were exclusion criteria for enrolment 
in both CESAR and EOLIA. The indication to initiate ECMO should, therefore, be carefully evaluated in these situations. Lastly, ECMO should be used in experienced centres and only after proven conventional management of severe ARDS (including lung protective mechanical ventilation and prone positioning) have been applied and failed [42], except when hypoxemia is immediately life-threatening, or when the patient is too unstable for prone positioning [43].

In conclusion, this meta-analysis of individual patient data of the CESAR and EOLIA trials showed strong evidence of a clinically meaningful benefit of early ECMO in severe ARDS patients. Another large study of ECMO appears unlikely in this setting and future research should focus on the identification of patients most likely to benefit from ECMO and optimization of treatment strategies after ECMO initiation [44].

The study was supported by the Direction de la Recherche Clinique et de l'Innovation (DRCI), Assistance Publique-Hopitaux de Paris (APHP), with a grant from the French Ministry of Health (CRC 2018, $\# 18,021$ ).

The EOLIA trial was supported by the Direction de la Recherche Clinique et du Développement (DRCD), Assistance Publique-Hôpitaux de Paris (APHP), with a grant from the French Ministry of Health (Programme Hospitalier de Recherche Clinique number, PHRC 2009 081,224), the EOLIA Trial Group, the Réseau Européen en Ventilation Artificielle (REVA) and the International ECMO Network (ECMONet, https://www.internatio nalecmonetwork.org).

The CESAR trial was supported by the UK NHS Health Technology Assessment, English National Specialist Commissioning Advisory Group, Scottish Department of Health, and Welsh Department of Health.

See the Supplement for the list of EOLIA and CESAR collaborators.

\section{Electronic supplementary material}

The online version of this article (https://doi.org/10.1007/s00134-020-06248-3) contains supplementary material, which is available to authorized users.

\footnotetext{
Author details

1 Sorbonne Université, INSERM, UMRS_1166-ICAN, Institute of Cardiometabolism and Nutrition, 75013 Paris, France. ${ }^{2}$ Service de médecine Intensive-réanimation, Hôpital Pitié-Salpêtrière, Assistance Publique-Hôpitaux de Paris, Sorbonne Université INSERM, UMRS_1166-ICA, Institute of Cardiometabolism and Nutrition, 47, boulevard de l'Hôpital, N75013 Paris, France. ${ }^{3}$ Congenital Heart Center, Shands Children's Hospital, University of Florida, Gainesville, FL 32610, USA. ${ }^{4}$ Sorbonne Université, INSERM, Institut Pierre Louis d'Epidémiologie et de Santé Publique, AP-HP.Sorbonne Université, Hôpital Pitié Salpêtrière, Département de Santé Publique, Centre de Pharmacoépidémiologie (Cephepi), CIC-1421, 75013 Paris, France. ${ }^{5}$ Birmingham Clinical Trials Unit, University of Birmingham, Edgbaston B15 2TT, UK. ${ }^{6}$ Columbia University College of Physicians and Surgeons/New York-Presbyterian Hospital, New York, NY, USA. ${ }^{7}$ Center for Acute Respiratory Failure, Columbia University Medical
}

Center, New York, NY, USA. ${ }^{8}$ Medical Statistics Department, London School of Hygiene and Tropical Medicine, Keppel Street, London WC1E 7HT, UK.

\section{Acknowledgements}

We thank Mrs Elizabeth Allen for her help in preparing the data of the CESAR trial.

\section{Compliance with ethical standards}

\section{Conflicts of interest}

Alain Combes reports grants from Getinge, personal fees from Getinge, Baxter and Xenios outside the submitted work. Matthieu Schmidt reports lecture fees from Getinge, Drager and Xenios outside the submitted work. The other authors declare that they have no conflicts of interest related to the purpose of this manuscript. The study protocol for the systematic review and IPD meta-analysis was approved by the relevant independent ethics committees: in France, Comité de Protection des Personnes CPP Ile de France VI, PitiéSalpêtrière, on 04/19/2018, Ref \#12 and in the UK by the Ethics committee of the London School of Hygiene and Tropical Medicine, on 04/12/2019, LSHTM Ethics Ref: 17159. Only patient characteristics and outcomes already evaluated in the trials were combined in this systematic review and meta-analysis.

\section{Publisher's Note}

Springer Nature remains neutral with regard to jurisdictional claims in published maps and institutional affiliations.

Received: 17 July 2020 Accepted: 15 September 2020 Published online: 6 October 2020

\section{References}

1. Brower RG, Matthay MA, Morris A, Schoenfeld D, Thompson BT, Wheeler A (2000) Ventilation with lower tidal volumes as compared with traditional tidal volumes for acute lung injury and the acute respiratory distress syndrome. N Engl J Med 342:1301-1308

2. Briel M, Meade M, Mercat A, Brower RG, Talmor D, Walter SD, Slutsky AS, Pullenayegum E, Zhou Q, Cook D, Brochard L, Richard JC, Lamontagne F, Bhatnagar N, Stewart TE, Guyatt G (2010) Higher vs lower positive end-expiratory pressure in patients with acute lung injury and acute respiratory distress syndrome: systematic review and meta-analysis. JAMA 303:865-873

3. Franchineau G, Brechot N, Lebreton G, Hekimian G, Nieszkowska A, Trouillet JL, Leprince P, Chastre J, Luyt CE, Combes A, Schmidt M (2017) Bedside contribution of electrical impedance tomography to setting positive end-expiratory pressure for extracorporeal membrane oxygenationtreated patients with severe acute respiratory distress syndrome. Am J Respir Crit Care Med 196:447-457

4. Papazian L, Forel JM, Gacouin A, Penot-Ragon C, Perrin G, Loundou A, Jaber S, Arnal JM, Perez D, Seghboyan JM, Constantin JM, Courant P, Lefrant JY, Guerin C, Prat G, Morange S, Roch A (2010) Neuromuscular blockers in early acute respiratory distress syndrome. N Engl I Med 363:1107-1116

5. Guerin C, Reignier J, Richard JC, Beuret P, Gacouin A, Boulain T, Mercier E, Badet M, Mercat A, Baudin O, Clavel M, Chatellier D, Jaber S, Rosselli S, Mancebo J, Sirodot M, Hilbert G, Bengler C, Richecoeur J, Gainnier M, Bayle F, Bourdin G, Leray V, Girard R, Baboi L, Ayzac L (2013) Prone positioning in severe acute respiratory distress syndrome. N Engl J Med 368:2159-2168

6. Bellani G, Laffey JG, Pham T, Fan E, Brochard L, Esteban A, Gattinoni L, van Haren F, Larsson A, McAuley DF, Ranieri M, Rubenfeld G, Thompson BT, Wrigge H, Slutsky AS, Pesenti A (2016) Epidemiology, patterns of care, and mortality for patients with acute respiratory distress syndrome in intensive care units in 50 countries. JAMA 315:788-800

7. Cummings MJ, Baldwin MR, Abrams D, Jacobson SD, Meyer BJ, Balough EM, Aaron JG, Claassen J, Rabbani LE, Hastie J, Hochman BR, SalazarSchicchi J, Yip NH, Brodie D, O'Donnell MR, (2020) Epidemiology, clinical course, and outcomes of critically ill adults with COVID-19 in New York City: a prospective cohort study. Lancet 395(10239):1763-1770. https:// doi.org/10.1016/S0140-6736(20)31189-2 
8. Yang X, Yu Y, Xu J, Shu H, Xia J, Liu H, Wu Y, Zhang L, Yu Z, Fang M, Yu T, Wang Y, Pan S, Zou X, Yuan S, Shang Y (2020) Clinical course and outcomes of critically ill patients with SARS-CoV-2 pneumonia in Wuhan, China: a single-centered, retrospective, observational study. Lancet Respiratory Med 8:475-481

9. Wang D, Hu B, Hu C, Zhu F, Liu X, Zhang J, Wang B, Xiang H, Cheng Z, Xiong Y, Zhao Y, Li Y, Wang X, Peng Z (2020) Clinical characteristics of 138 hospitalized patients with 2019 novel coronavirus-infected pneumonia in Wuhan, China. JAMA 323:1061-1069

10. Brodie D, Slutsky AS, Combes A (2019) Extracorporeal life support for adults with respiratory failure and related indications: a review. JAMA 322:557-568

11. Schmidt M, Pham T, Arcadipane A, Agerstrand C, Ohshimo S, Pellegrino V, Vuylsteke A, Guervilly C, McGuinness S, Pierard S, Breeding J, Stewart C, Ching SSW, Camuso JM, Stephens RS, King B, Herr D, Schultz MJ, Neuville M, Zogheib E, Mira JP, Roze H, Pierrot M, Tobin A, Hodgson C, Chevret S, Brodie D, Combes A (2019) Mechanical ventilation management during extracorporeal membrane oxygenation for acute respiratory distress syndrome. an international multicenter prospective cohort. Am J Respir Crit Care Med 200:1002-1012

12. Pham T, Combes A, Roze H, Chevret $\mathrm{S}$, Mercat A, Roch A, Mourvillier B, Ara-Somohano C, Bastien O, Zogheib E, Clavel M, Constan A, Marie Richard JC, Brun-Buisson C, Brochard L (2013) Extracorporeal membrane oxygenation for pandemic influenza $A(\mathrm{H} 1 \mathrm{~N} 1)$-induced acute respiratory distress syndrome: a cohort study and propensity-matched analysis. Am J Respir Crit Care Med 187:276-285

13. Davies A, Jones D, Bailey M, Beca J, Bellomo R, Blackwell N, Forrest $P$, Gattas D, Granger E, Herkes R, Jackson A, McGuinness S, Nair P, Pellegrino V, Pettila V, Plunkett B, Pye R, Torzillo P, Webb S, Wilson M, Ziegenfuss M (2009) Extracorporeal membrane oxygenation for 2009 influenza A(H1N1) acute respiratory distress syndrome. JAMA 302:1888-1895

14. Noah MA, Peek GJ, Finney SJ, Griffiths MJ, Harrison DA, Grieve R, Sadique MZ, Sekhon JS, MCAuley DF, Firmin RK, Harvey C, Cordingley JJ, Price S, Vuylsteke A, Jenkins DP, Noble DW, Bloomfield R, Walsh TS, Perkins GD, Menon D, Taylor BL, Rowan KM (2011) Referral to an extracorporeal membrane oxygenation center and mortality among patients with severe 2009 influenza A(H1N1). JAMA 306:1659-1668

15. Peek GJ, Mugford M, Tiruvoipati R, Wilson A, Allen E, Thalanany MM, Hibbert CL, Truesdale A, Clemens F, Cooper N, Firmin RK, Elbourne D (2009) Efficacy and economic assessment of conventional ventilatory support versus extracorporeal membrane oxygenation for severe adult respiratory failure (CESAR): a multicentre randomised controlled trial. Lancet 374:1351-1363

16. Peek GJ, Elbourne D, Mugford M, Tiruvoipati R, Wilson A, Allen E, Clemens F, Firmin R, Hardy P, Hibbert C, Jones N, Killer H, Thalanany M, Truesdale A (2010) Randomised controlled trial and parallel economic evaluation of conventional ventilatory support versus extracorporeal membrane oxygenation for severe adult respiratory failure (CESAR). Health Technol Assessment (Winchester, England) 14:1-46

17. Combes A, Hajage D, Capellier G, Demoule A, Lavoue S, Guervilly C, Da Silva D, Zafrani L, Tirot P, Veber B, Maury E, Levy B, Cohen Y, Richard C, Kalfon P, Bouadma L, Mehdaoui H, Beduneau G, Lebreton G, Brochard L, Ferguson ND, Fan E, Slutsky AS, Brodie D, Mercat A (2018) Extracorporeal membrane oxygenation for severe acute respiratory distress syndrome. $N$ Engl J Med 378:1965-1975

18. Bernard GR, Artigas A, Brigham KL, Carlet J, Falke K, Hudson L, Lamy M, Legall JR, Morris A, Spragg R (1994) The American-European consensus conference on ARDS. Definitions, mechanisms, relevant outcomes, and clinical trial coordination. Am J Respir Crit Care Med 149:818-824

19. Ranieri VM, Rubenfeld GD, Thompson BT, Ferguson ND, Caldwell E, Fan E, Camporota L, Slutsky AS (2012) Acute respiratory distress syndrome: the Berlin Definition. JAMA 307:2526-2533

20. Combes A, Brodie D, Bartlett R, Brochard L, Brower R, Conrad S, De Backer D, Fan E, Ferguson N, Fortenberry J, Fraser J, Gattinoni L, Lynch W, MacLaren G, Mercat A, Mueller T, Ogino M, Peek G, Pellegrino V, Pesenti A, Ranieri M, Slutsky A, Vuylsteke A (2014) Position paper for the organization of extracorporeal membrane oxygenation programs for acute respiratory failure in adult patients. Am J Respir Crit Care Med 190:488-496

21. Combes A, Pesenti A, Ranieri VM (2017) Fifty years of research in ARDS. Is extracorporeal circulation the future of acute respiratory distress syndrome management? Am J Respir Crit Care Med 195:1161-1170
22. Sterne JAC, Savovic J, Page MJ, Elbers RG, Blencowe NS, Boutron I, Cates CJ, Cheng HY, Corbett MS, Eldridge SM, Emberson JR, Hernan MA, Hopewell S, Hrobjartsson A, Junqueira DR, Juni P, Kirkham JJ, Lasserson T, Li T, McAleenan A, Reeves BC, Shepperd S, Shrier I, Stewart LA, Tilling K, White IR, Whiting PF, Higgins JPT (2019) RoB 2: a revised tool for assessing risk of bias in randomised trials. BMJ 366:14898

23. Tierney JF, Stewart LA, Clarke M (2019) Chapter 26: Individual participant data. In: Higgins JPT, Thomas J, Chandler J, Cumpston M, LiT, Page MJ, Welch VA (eds) Cochrane handbook for systematic reviews of interventions version 6-0 (updated July 2019) Cochrane, www.training.cochrane. org/handbook

24. Li X, Scales DC, Kavanagh BP (2018) Unproven and expensive before proven and cheap: extracorporeal membrane oxygenation versus prone position in acute respiratory distress syndrome. Am J Respir Crit Care Med 197:991-993

25. Schmidt M, Combes A, Shekar K (2019) ECMO for immunosuppressed patients with acute respiratory distress syndrome: drawing a line in the sand. Intensive Care Med 45:1140-1142

26. Fernando SM, Qureshi D, Tanuseputro P, Fan E, Munshi L, Rochwerg B, Talarico R, Scales DC, Brodie D, Dhanani S, Guerguerian AM, Shemie SD, Thavorn K, Kyeremanteng K (2019) Mortality and costs following extracorporeal membrane oxygenation in critically ill adults: a populationbased cohort study. Intensive Care Med 45:1580-1589

27. Fan E, Karagiannidis C (2019) Less is more: not (always) simple-the case of extracorporeal devices in critical care. Intensive Care Med 45:1451-1453

28. Zapol WM, Snider MT, Hill JD, Fallat RJ, Bartlett RH, Edmunds LH, Morris AH, Peirce EC 2nd, Thomas AN, Proctor HJ, Drinker PA, Pratt PC, Bagniewski A, Miller RG Jr (1979) Extracorporeal membrane oxygenation in severe acute respiratory failure. A randomized prospective study JAMA 242:2193-2196

29. Morris AH, Wallace CJ, Menlove RL, Clemmer TP, Orme JF Jr, Weaver LK, Dean NC, Thomas F, East TD, Pace NL et al (1994) Randomized clinical trial of pressure-controlled inverse ratio ventilation and extracorporeal $\mathrm{CO} 2$ removal for adult respiratory distress syndrome. Am J Respir Crit Care Med 149:295-305

30. Goligher EC, Tomlinson G, Hajage D, Wijeysundera DN, Fan E, Juni P, Brodie D, Slutsky AS, Combes A (2018) Extracorporeal membrane oxygenation for severe acute respiratory distress syndrome and posterior probability of mortality benefit in a post hoc bayesian analysis of a randomized clinical trial. JAMA 320:2251-2259

31. Aoyama H, Uchida K, Aoyama K, Pechlivanoglou P, Englesakis M, Yamada Y, Fan E (2019) Assessment of therapeutic interventions and lung protective ventilation in patients with moderate to severe acute respiratory distress syndrome: a systematic review and network meta-analysis. JAMA network open 2:e198116

32. Munshi L, Walkey A, Goligher E, Pham T, Uleryk EM, Fan E (2019) Venovenous extracorporeal membrane oxygenation for acute respiratory distress syndrome: a systematic review and meta-analysis. The Lancet Respiratory medicine 7:163-172

33. Tierney JF, Vale C, Riley R, Smith CT, Stewart L, Clarke M, Rovers M (2015) Individual participant data (IPD) meta-analyses of randomised controlled trials: guidance on their use. PLoS Med 12:e1001855

34. Tierney JF, Fisher DJ, Burdett S, Stewart LA, Parmar MKB (2020) Comparison of aggregate and individual participant data approaches to meta-analysis of randomised trials: an observational study. PLoS Med 17:e1003019

35. Abrams D, Schmidt M, Pham T, Beitler JR, Fan E, Goligher EC, McNamee JJ, Patroniti N, Wilcox ME, Combes A, Ferguson ND, McAuley DF, Pesenti A, Quintel M, Fraser J, Hodgson CL, Hough CL, Mercat A, Mueller T, Pellegrino V, Ranieri VM, Rowan K, Shekar K, Brochard L, Brodie D (2020) Mechanical ventilation for acute respiratory distress syndrome during extracorporeal life support. research and practice. Am J Respir Crit Care Med 201:514-525

36. Serpa Neto A, Deliberato RO, Johnson AEW, Bos LD, Amorim P, Pereira SM Cazati DC, Cordioli RL, Correa TD, Pollard TJ, Schettino GPP, Timenetsky KT, Celi LA, Pelosi P, Gama de Abreu M, Schultz MJ (2018) Mechanical power of ventilation is associated with mortality in critically ill patients: an analysis of patients in two observational cohorts. Intensive Care Med 44:1914-1922

37. World Health Organization: Clinical management of severe acute respiratory infection when COVID-19 disease is suspected. Last accessed July, 
10 2020: https://www.who.int/publications/i/item/clinical-management -of-covid-19

38. Alhazzani W, Møller MH, Arabi YM, Loeb M, Gong MN, Fan E, Oczkowski S, Levy MM, Derde L, Dzierba A, Du B, Aboodi M, Wunsch H, Cecconi M, Koh Y, Chertow DS, Maitland K, Alshamsi F, Belley-Cote E, Greco M, Laundy M, Morgan JS, Kesecioglu J, McGeer A, Mermel L, Mammen MJ, Alexander PE, Arrington A, Centofanti JE, Citerio G, Baw B, Memish ZA, Hammond N, Hayden FG, Evans L, Rhodes A (2020) Surviving sepsis campaign: guidelines on the management of critically ill adults with coronavirus disease 2019 (COVID-19). Intensive Care Med 46:854-887

39. Schmidt M, Hajage D, Lebreton G, Monsel A, Voiriot G, Levy D, Baron E, Beurton A, Chommeloux J, Meng P, Nemlaghi S, Bay P, Leprince P, Demoule A, Guidet B, Constantin JM, Fartoukh M, Dres M, Combes A (2020) Extracorporeal membrane oxygenation for severe acute respiratory distress syndrome associated with COVID-19: a retrospective cohort study. Lancet Respiratory Med. https://doi.org/10.1016/S2213 $-2600(20) 30328-3$

40. Fisher DJ, Carpenter JR, Morris TP, Freeman SC, Tierney JF (2017) Metaanalytical methods to identify who benefits most from treatments: daft, deluded, or deft approach? BMJ 356:j573
41. Schmidt M, Zogheib E, Roze H, Repesse X, Lebreton G, Luyt CE, Trouillet JL, Brechot N, Nieszkowska A, Dupont H, Ouattara A, Leprince P, Chastre J, Combes A (2013) The PRESERVE mortality risk score and analysis of longterm outcomes after extracorporeal membrane oxygenation for severe acute respiratory distress syndrome. Intensive Care Med 39:1704-1713

42. Abrams D, Ferguson ND, Brochard L, Fan E, Mercat A, Combes A, Pellegrino V, Schmidt M, Slutsky AS, Brodie D (2019) ECMO for ARDS: from salvage to standard of care? Lancet Respiratory Med 7:108-110

43. MacLaren G, Combes A, Brodie D (2020) Saying no until the moment is right: initiating ECMO in the EOLIA era. Intensive Care Med. https://doi. org/10.1007/s00134-020-06185-1

44. Guervilly C, Prud'homme E, Pauly V, Bourenne J, Hraiech S, Daviet F, Adda M, Coiffard B, Forel JM, Roch A, Persico N, Papazian L (2019) Prone positioning and extracorporeal membrane oxygenation for severe acute respiratory distress syndrome: time for a randomized trial? Intensive Care Med 45:1040-1042 